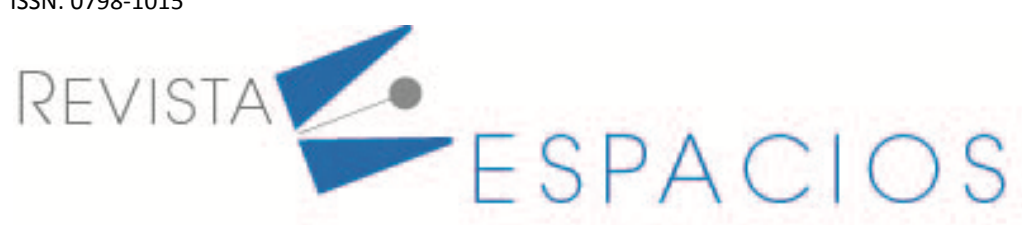

\title{
Influencia del género en los factores de trastornos del juego
}

\section{Influence of gender on gambling disorder factors}

\author{
LLERENA ALCÁNTARA, Isabel M. ${ }^{1}$ \\ MARÍN MARÍN, José A. ${ }^{2}$ \\ HERNÁNDEZ GARCÍA, María ${ }^{3}$ \\ MORENO GUERRERO, Antonio J. ${ }^{4}$
}

\begin{abstract}
Resumen
El objetivo de este estudio es conocer el nivel de trastorno del juego en estudiantes desde secundaria obligatoria hasta universitarios a partir de la variable género. La metodología de investigación ha sido cuantitativa de corte descriptivo y correlacional, haciendo uso de un diseño no experimental del paradigma transeccional. El instrumento usado es el cuestionario de Internet Gaming Disorder-20 (IGC-20). Los resultados muestran que los hombres y los menores de 24 años son los que presentan mayores valores de adicción al juego.

Palabras clave: adicción, joven, género, trastornos del juego

Abstract

The aim of this study is to find out the level of gambling disorder in students from compulsory secondary school to university from the gender variable. The research methodology was quantitative, descriptive and correlational, using a non-experimental design with a cross-sectional paradigm. The instrument used was the Internet Gaming Disorder-20 (IGC-20) questionnaire. The results show that men and those under 24 years of age are those with the highest levels of gambling addiction.

Key words: addiction, youth, gender, gambling disorders
\end{abstract}

\section{Introducción}

Hoy día se sabe que Internet es una herramienta esencial en la vida cotidiana dado que sirve como fuente de relajación, diversión y socialización, entre otros aspectos (Bouna, Muhle, Kornhuber y Lenz, 2015; MorenoGuerrero, 2019).

Existe una gran diversidad de jóvenes y adultos que usan las tecnologías de la información y comunicación (TIC) para evadirse de la realidad (Hui, Wu \& Pun, 2019), relacionándose de una forma problemática o incluso desarrollando adicción a, por ejemplo, internet o teléfonos inteligentes, entre otras (Chen et al, 2020; RodríguezGarcía, Moreno-Guerrero y López, 2020; Trujillo Torres et al., 2014). Este problema puede afectar al entorno social de los individuos así como la relación con su propia familia (Adams et al, 2018; Bonnaire et al., 2019),

\footnotetext{
${ }^{1}$ Estudiante Máster de Profesorado. Universidad de Granada. España. imllere@correo.ugr.es

2 Profesor e investigador. Departamento de Didáctica y Organización Escolar. Universidad de Granada. España. jmarin@ugr.es

${ }^{3}$ Estudiante Máster de Atención a la Diversidad. Universidad de Granada. España. ghmariahg@correo.ugr.es

${ }^{4}$ Profesor e investigador. Departamento de Didáctica y Organización Escolar. Universidad de Granada. España. ajmoreno@ugr.es
} 
incluso poner en riesgo la salud de las personas (Kim et al., 2019; Kircaburun, Griffiths \& Billieux, 2019; Müller et al., 2019),

Por ello, es fundamental que se sepa controlar las situaciones en las que se desarrolla el juego, ya que puede llegar a ser excesivo y problemático (Zhang et al., 2015; Chen et al., 2020). El juego se asocia a un mayor bienestar y desarrollo de diversas habilidades cognitivas, siempre que se utilice de forma moderada (Loton \& Lubman, 2016).

La adicción al juego virtual se conoce como IGD (Internet Gaming Disorder ) y se entiende como un uso excesivo de Internet, juego o teléfonos inteligentes durante mucho tiempo (Jo et al., 2019; Chen et al., 2020), ya sea en línea (Kim et al., 2018) o fuera de línea (Lemmens y Hendriks, 2016), que llegan a provocar efectos negativos no deseados en los sujetos (Borges et al., 2019) y para su familias (González et al., 2018; Schneider, King \& Delfabbro, 2017). Estos síntomas pueden ir de más leves (llegar tarde) a bastante graves (perder el trabajo) (Eichenbaum et al., 2015). Los sujetos con IGD se caracterizan por tener una imagen negativa de sí mismo, tanto ideal como real, por lo que debe ser notado y aplicado con una terapia apropiada (Kim et al., 2018). Existe más riesgo de producirse cuando la satisfacción de las tres necesidades básicas -competencia, autonomía y parentesco- es baja en el mundo real y alta en los videojuegos (Allen y Anderson, 2018).

Al mismo tiempo pueden alcanzar niveles patológicos (Bouna et al., 2015) y, a veces, los individuos no se dan cuenta de las consecuencias graves que esto tiene para ellos mismos (Wang et al., 2019). Asimismo, muestran una disminución del control ejecutivo (Liu et al., 2018), así como del cognitivo, (Liu et al., 2018; Wang et al., 2018) y una mayor susceptibilidad a las señales del juego (Wang et al., 2018) mientras pasan el tiempo con juegos de diversa índole en Internet.

\subsection{Revisión de la literatura}

El IGD está incluido en el Manual de Diagnóstico y Estadístico de los Trastornos Mentales, Quinta Edición (DSM5) (American Psychiatric Association, 2018), que integra los criterios de su diagnóstico (Cai et al., 2016; Fuster et al., 2016; Jo et al., 2019; Ko et al., 2019; Shin et al, 2019; Zajac, Ginley y Chang, 2019), en los que hay nueve criterios de básicos (Müller et al, 2019) y cinco criterios de corte (Kim et al., 2016; Sprong et al., 2019). Además, está incluido en la undécima revisión final de la Clasificación Internacional de Enfermedades (CIE-11), entendiéndose como un código de diagnóstico (Jo et al., 2019; Wartberg, Kriston \& Thomasius, 2020), siendo reconocido por primera vez en mayo de 2013 (Ji, Hsieh \& Hsiao, 2016).

El IGD se centra en los juegos de azar por Internet y en aquellos adaptados para el uso de las redes sociales (Bouna et al., 2018). Según diversos estudios, los criterios aplicados están altamente relacionados con el tiempo que se les dedica a las actividades de Internet. Los criterios proporcionados resultaron ser fáciles de usar, comprensibles y bien aceptados. Por ello, los resultados contribuyen a una mejor comprensión de los juegos patológicos en Internet y el uso de las redes sociales y, asimismo, aportan pruebas de que los marcadores biológicos de los trastornos por consumo de sustancias están implicados en la adicción a Internet.

En conjunto, los diversos síntomas del IGD tienen diferente peso clínico. Cada uno de ellos puede evidenciar funciones diagnósticas únicas en el desarrollo de los juegos adictivos (Pontes et al., 2019), más concretamente, aquellos que son de estrategias en tiempo real, juegos de rol y de fantasía (Eichenbaum et al., 2015).

Algunos de los síntomas pueden ser la tolerancia, el ansia, modificación del estado de ánimo, la prominencia, la soledad, impulsividad (Chen et al., 2016; Du et al., 2016; Dang et al., 2019; Müller et al., 2019; Shin et al., 2019; Yen et al., 2019), alteraciones de las funciones cognitivas y sociales (Park et al., 2016), desapego (Laier, Wegmann \& Brand, 2018), problemas en la regulación de emociones (Kang et al., 2018) y la falta de atención (Du et al., 2016), siendo este último un factor muy influyente en el IGD (Hsieh \& Hsiao, 2016). Además, se le asocian otros 
síntomas como la depresión (Jeong et al., 2019, Kircaburun et al., 2019), el número de horas de juego y a la negación, siendo estos dos últimos los predictores más fuerte de los síntomas (Schneider et al., 2017). Por otro lado, pueden presentar una baja resistencia, alto estrés o una baja capacidad para superar cualquier situación traumática (Yen et al., 2019). A pesar de todo esto, en la actualidad, no se tienen pruebas de que el IGD tenga un fundamento neurobiológico como trastorno adictivo (Kim et al., 2019).

Los sujetos con IGD pueden estar impedidos en algunos aspectos de sus funciones de inhibición y de toma de decisiones (Wang et al., 2018), ya que este último aspecto está vinculado a un problema específico con las pistas del juego (Yao et al., 2015). Al mismo tiempo puede dar lugar a que dichos sujetos tengan alteraciones del sueño así como un bajo rendimiento académico.

Los predictores fundamentales de dicho trastorno incluyen rasgos de la personalidad neurótica e introvertida, y la motivación relacionada con los logros (Carlisle et al., 2019). También cuenta con una mayor proporción en el género masculino (Fam, 2018), ya que suelen jugar mucho más a los videojuegos (Dong et al., 2018), con una relación de hombres y mujeres de 3:1 (Dong et al., 2018; Wang et al., 2019). Esta proporción se observa también en la etapa infantil, donde se observa ya dicha proporción (Paulus et al., 2017). Los estudios plantean que ellas tienen un mejor control ejecutivo que los hombres al enfrentarse a las señales de juego, por lo que se resisten contra el desarrollo del IGD (Dong et al., 2018).

Según diversos estudios, el IGD presenta relación con la alexitimia (Bonnaire y Baptista, 2019), es decir, la incapacidad de identificar las emociones, por lo que tienden a ser agresivos entre los compañeros o personas (Evren et al., 2018), precisando de intervenciones terapéuticas más activas (Lee et al, 2018). La agresión puede desarrollarse de forma más fácil y severa mediante juegos que sean complejos, interminables y de impulso social, independientemente de las peculiaridades a cada persona (King et al., 2019).

También tiene relación con el Trastorno por Déficit de Atención con Hiperactividad (TDAH) (Evren et al., 2018), aunque, hasta la fecha, hay pocos estudios que hablan de sus asociaciones (Marmet, Studer, Grazioli y Gmel, 2018; Romero-Rodríguez et al., 2021), estando más relacionado con la falta de atención.

Sobre la base de los criterios del IGD, el cuestionario más usado para evaluarlo es el de la Escala de Trastorno de Juegos en Internet con nueve elementos (IGDS-SF9) (Pontes \& Griffiths, 2016; Evren et al., 2018; Gómez et al., 2019; Evren et al., 2018; Chen et al., 2020; Leung et al., 2020). Además de otras escalas psicométricas breves tales como la Escala de Adicción Basada en Aplicaciones para Teléfonos Inteligentes (SABAS) compuesto por seis elementos y la Escala de Adicción a los Medios Sociales de Bergen (BSMAS) formada por seis también (Chen et al., 2020; Leung et al., 2020).

Estas escalas han examinado a sujetos en un periodo corto, por ejemplo dos semanas, y no se sabe con claridad si pueden ser invariables si se lleva a ampliar dicho periodo, como puede ser tres meses (Chen et al., 2020). Además, se determina que son instrumentos válidos para evaluar correctamente las adicciones relacionadas con Internet, siendo usados por los profesionales de la salud (Leung et al., 2020).

Por otro lado, según diversas investigaciones (Fuster et al., 2016; Romero-Rodríguez et al., 2021), se concluye que la versión española del test IGD-20 es viable y fiable, pudiendo ser usada en el estudio del IGD de aquellas poblaciones con habla hispana.

Para el IGD se han hecho otras investigaciones, como por ejemplo las correlaciones psicosociales y la comorbilidad (Cheng, Cheunf \& Wang, 2018; Liu et al., 2018). No hay gran cantidad de investigaciones sobre los mecanismos de desarrollo y los procesos que conducen al trastorno (Throuvala et al., 2019). Sigue sin estar claro (Paulus et al., 2018) si dicho trastorno representa una adicción a Internet o si evalúa conductas específicas que se producen en el contexto de los videojuegos (Sprong et al., 2019). 
Aunque en los últimos años han crecido los estudios sobre el IGD (Torres et al., 2019), incluido los ensayos clínicos (Zajac et al., 2019), se sigue debatiendo acerca de los criterios respectivos que se tienen que utilizar, así como la condición de la afección como problema de salud mental (Kuss et al., 2018). Se dice que se sigue sin tener conclusiones sólidas y claras (Liu et al., 2015; Han et al., 2018).

Por ello, se espera que se desarrolle una mejor teoría, así como estudios metodológicos y pruebas empíricas más sólidos en relación a los juegos problemáticos y sus consecuencias en el impacto psicosocial (Griffiths et al., 2015). Todo esto debería favorecer a las descripciones clínicas de los juegos problemáticos de Internet y, a su vez, mejorar la cuantificación de la abstinencia de la IGD junto a los tratamientos (Kaptsis et al., 2016).

Debido al aumento del uso de las tecnologías por parte de los estudiantes en distintos ámbitos de su vida diaria, social y familiar, y los diversos efectos que provocan en ellos, ya sean negativos o positivos, surge esta investigación para seguir descubriendo y ampliando el campo de conocimiento acerca de este trastorno.

El objetivo principal que se plantea es conocer el nivel de trastorno del juego a través de Internet en estudiantes de diferentes etapas educativas. Este objetivo general conduce a la formulación de los siguientes objetivos específicos, con el propósito de guiar la investigación:

- $\quad$ Determinar el nivel de trastorno del juego según las distintas variables de estudio.

- $\quad$ Determinar las dimensiones más y menos afectadas del trastorno del juego en estudiantes.

- Identificar la relación existente entre el género y los trastornos del juego en discentes.

A raíz de estos objetivos surge la siguiente pregunta de investigación:

- $\quad$ PI: ¿Cómo afecta el género en los trastornos del juego a través de Internet en los discentes?

\section{Metodología}

\subsection{Diseño de investigación y análisis de datos}

Este estudio se ha centrado en una metodología de investigación cuantitativa de corte descriptivo y correlacional, haciendo uso de un diseño no experimental de paradigma transeccional (Hernández, Fernández y Baptista, 2014).

Para la elaboración del análisis de los datos se ha aplicado unos estadísticos básicos tales como la media (M) y la desviación típica (DT) y, al mismo tiempo, pruebas específicas para formar la propensión de la distribución como el coeficiente de asimetría de Pearson (ASI) y el de apuntamiento de Fisher (CUR). Asimismo para establecer la correspondencia entre las variables se ha utilizado el Test Chi-cuadrado de Pearson ( $\chi 2$ ), junto con la $\vee$ de Cramer (V) y el coeficiente de contingencia (Cont.) para saber cuál es la fuerza de relación entre ellas.

\subsection{Participantes}

Un total de 180 estudiantes han conformado la muestra de este estudio, de los cuales el $47.2 \%$ son hombres y el resto mujeres, teniendo una edad media de 21 años ( $D T=1.749)$. Los estudiantes pertenecen a diversos periodos educativos, entre los que se encuentran un $10.56 \%$ de estudiantes de la Educación Secundaria Obligatoria (ESO), un $12.78 \%$ a Bachiller, un $43.33 \%$ de Formación Profesional (FP) y un 33.33\% de Educación Superior.

La composición de la muestra se ha elaborado a través de un muestreo no probabilístico por conveniencia, ya que se ha utilizado el periodo lectivo para pasar el cuestionario y, así como, por las técnicas de la bola de nieve, haciendo uso de redes sociales (Facebook, Twitter y Linkedln) y otras medios tecnológicos de comunicación, tales como Telegram y WhatsApp. 


\subsection{Instrumento}

Para la recopilación de datos se ha empleado el cuestionario de Internet Gaming Disorder-20 (IGC-20) elaborado por Fuster, Carbonell, Pontes y Griffiths (2016). El instrumento está compuesto por un total de 23 ítems, distribuidos en distintas dimensiones: a) Dimensión socioeducativa (3 ítems); b) Dimensión prominencia (3 ítems); c) Dimensión modificación del estado de ánimo (3 ítems); e) Dimensión tolerancia (3 ítems); f) Dimensión síntomas de abstinencia (3 ítems); g) Dimensión conflicto (5 ítems); h) Dimensión recaída (3 ítems).

La mayoría de las preguntas del cuestionario están configuradas en escala Likert de 5 puntos (1=Totalmente en desacuerdo; 2=En desacuerdo; 3=Ni de acuerdo ni en desacuerdo; 4=De Acuerdo; 5=Totalmente de acuerdo), aunque también se hayan otras cuestiones de respuesta cerrada y dicotómicas.

El procedimiento metodológico que se ha seguido para la validación y fiabilidad del instrumento es realizado por Fuster et al (2016). El cual ha llevado a cabo un análisis de la distancia de Mahalanobis con el propósito de identificar los valores atípicos multivariantes dentro de la muestra y se hace uso del valor umbral de $p<0.001$ (Tabachnick y Fidell, 2007).

Las pruebas de normalidad univariante de Shapiro-Wilk, las cuales proponen una suposición moderada de normalidad para las variables involucrada en el análisis. Para validar el idioma español de versión de la prueba IGD-20, se hizo un CFA, utilizando el máximo de Estimación de la verosimilitud con error estándar robusto (MLR) en MPLUS 6.12 (Muthen y Muthen, 2011).

Los indicadores evaluados de ajuste integran el valor $p$ de Chi Cuadrado mayor de 0.05, el ajuste comparativo (CFI), el índice de ajuste de Tucker Lewis (TLI), y el Root Mean Error Cuadrado de Aproximación (RMSEA). Se puede decir que existe un ajuste apropiado para el CFI y el TLI, siendo mayores de 0.90, y el RMSEA es menor que el 0.06 .

Con esto se puede confirmar que las clases identificadas hicieron un análisis de perfil latente (LPA), usando MPLUS 6.12. Al utilizar dicho análisis (LPA), se tuvieron en cuenta diversas consideraciones estadísticas y teóricas para conseguir un mejor ajuste de los datos para la caracterización de grupos de personas con respuestas parecidas para ciertas variables.

EI LPA es un modelo que permite identificar subgrupos, tipos o categorías de individuos de la población en estudio e identificar patrones de respuesta basados en características observadas, relacionándolos con un conjunto de clases latentes. En este sentido, la variable latente, o constructo, no se observa directamente, sino que se mide indirectamente a través de dos o más variables observadas con la máxima estimación probabilística.

La probabilidad de que un sujeto esté correctamente clasificado se debe estimar, de forma simultánea, con el modelo general. Por lo que el análisis realizado tuvo en cuenta el Criterio de Información de Akaike (AIC) y el Criterio Bayesiano Criterio de Información (BIC) y, al mismo tiempo, el Tamaño de la Muestra Ajustado BIC (SSABIC).

Otra prueba, conocida como la probabilidad ajustada (LoMendell-Rubin Adjusted LRT TEST), se usó para ver el grado de unión entre dos modelos anidados que se distinguen en una clase fijada. También se utilizó la estadística de entropía relativa, basada como un indicador de la bondad de la organización de los individuos según los valores aproximados a 1 , ya que estos significan una alta certeza en la misma.

Con todos estos modelos se ha analizado el modelo que se adapta mejor a los datos, considerándose el AIC, BIC y SSABIC más bajo. La prueba LMR y el valor relativo de entropía se mostraban para seleccionar el modelo más apropiado de dos o más modelos que compartían criterios de información parecida (AIC, BIC y SSABIC). Las 
variables latentes categóricas conseguidas son variables empíricas que simbolizan clases de sujetos que comparten resultados parecidos de la prueba IGD-20.

Además, se hizo un análisis de la curva ROC (característica operativa receptora), con el nombre de "jugadores desordenados", siendo representado como un patrón de oro para evaluar el instrumento que diferencia entre dos elementos en una clasificación binaria (jugadores desordenados y no desordenados).

Y el análisis de la curva ROC identificó los puntos de corte en relación a su sensibilidad (porcentaje de verdaderos positivos entre los jugadores desordenados) y su especificidad (porcentaje de verdaderos negativos entre los jugadores no desordenados).

En función a todas estas pruebas, los autores concluyeron que el instrumento presenta valores adecuados de validez y fiabilidad.

\subsection{Variables}

Para mejorar la presentación y facilitar la interpretación de los resultados, en este apartado se presentan las distintas variables junto con la codificación utilizada. Las variables consideradas como independiente en este estudio son: "A menudo pierdo horas de sueño debido a largas sesiones de juego (PRO_1)"; "Suelo pensar en mi próxima sesión de juego cuando no estoy jugando (POR_2)"; "Creo que jugar se ha convertido en la actividad que más tiempo consume mi vida (PRO_3)"; "Nunca juego a videojuegos para sentirme mejor (ANI_1)"; "Juego a videojuegos porque me ayuda a lidiar con los sentimientos desagradables que puedo tener (ANI_2)", "Juego a videojuegos para olvidar cosas que me preocupan (ANI_3)"; "Durante el último año he aumentado significativamente la cantidad de horas de juego a videojuegos (TOL_1)", "Necesito pasar cada vez más tiempo jugando a videojuegos (TOL_2)"; "A menudo pienso que un día entero no es suficiente para hacer todo lo necesito hacer en el juego (TOL_3)"; "Me siento más irritable cuando no estoy jugando (ABS_1)"; "Me siento triste si no puedo jugar a videojuegos (ABS_2)"; "Tiendo a ponerme nervioso si no puedo jugar por alguna razón (ABS_3)"; "He perdido interés en otras aficiones debido a jugar (CON_1)"; "He mentido a mis familiares debido a la cantidad de tiempo que dedico a videojuegos (CON_2)"; "Creo que jugar ha puesto en peligro la relación con mi pareja (CON_3)"; "Sé que mis actividades cotidianas no se han visto negativamente afectadas por jugar (CON_4)"; "Creo que jugar está afectando negativamente áreas importantes de mi vida (CON_5)"; " Me gustaría reducir mi tiempo de juego pero me resulta difícil (REC_1)"; "No creo que pudiera dejar de jugar (REC_2)"; "A menudo me propongo jugar menos pero acabo por no logarlo (REC_3)". Las variables consideradas como dependiente han sido género (G).

\subsection{Procedimiento}

El estudio empezó en enero de 2020 a través de la realización de la adaptación del instrumento validado al Formulario de Google. A continuación, se hizo el envío del instrumento mediante correo electrónico a diversos estudiantes tanto de distintos Institutos de Secundaria (I.E.S.) como de la Universidad de Granada (Campus de Ceuta) a finales del mes de enero. Además, el cuestionario se envió por las redes sociales (Facebook, Instagram, entre otros). El proceso de recogida de toda la información se finalizó el mes de abril del mismo año. Una vez terminado, se enviaron y adecuaron los datos al programa Statistical Package for the Social Sciences (SPSS) versión 25, para el análisis de las variables del estudio, y dar así respuesta a los objetivos y cuestiones planteadas en esta investigación. 


\section{Resultados}

\subsection{Análisis descriptivo}

Para dar respuesta a los objetivos planteados en la investigación, seguidamente se muestran los distintos resultados conseguidos. En términos generales se ve que hay diferencias de medias entre las variables en el que se muestra que no todos los ítems han sido valorados de la misma forma. La variable con más media es "Nunca juego a videojuegos para sentirme mejor (ANI_1)", y con menos media es "He mentido a mis familiares debido a la cantidad de tiempo que dedico a videojuegos (CON_2)". En la desviación típica se observa que no hay una tendencia clara de respuesta entre los encuestados, ya que aquellas que son realizadas por los estudiantes están dispersas. Por otro lado, los niveles de asimetría y curtosis son altos en diversas variables, mostrando que la distribución de respuesta anormal, lo que justifica la utilización de pruebas no paramétricas en el estudio (tabla 1).

La curtosis es platicúrtica en las variables "A menudo pierdo horas de sueño debido a largas sesiones de juego (PRO_1)", "Suelo pensar en mi próxima sesión de juego cuando no estoy jugando (PRO_2)", "Nunca juego a videojuegos para sentirme mejor (ANI_1)", "Juego a videojuegos porque me ayuda a lidiar con los sentimientos desagradables que puedo tener (ANI_2)", "Juego a videojuegos para olvidar cosas que me preocupan (ANI_3)", "Durante el último año he aumentado significativamente la cantidad de horas de juego a videojuegos (TOL_1)", "He perdido interés en otras aficiones debido a jugar (CON_1)", "He mentido a mis familiares debido a la cantidad de tiempo que dedico a jugar (CON_2)", "Sé que mis actividades cotidianas no se han visto negativamente afectadas por jugar (CON_4)", "Me gustaría reducir mi tiempo de juego pero me resulta difícil (REC_1)", "No creo que pudiera dejar de jugar (REC_2)", y "A mendo me propongo jugar menos pero acabo por no lograrlo" (REC_3)".

La curtosis es leptocúrtica en "Creo que jugar se ha convertido en la actividad que más tiempo consume mi vida" (PRO_3), "Necesito pasar cada vez más tiempo jugando a videojuegos (TOL_2)", "A menudo pienso que un día entero no es suficiente para hacer todo lo necesito hacer en el juego. (TOL_3)" y "Me siento triste si no puedo jugar a videojuegos (ABS_2)".

La curtosis es mesocúrtica en "Me siento más irritable cuando no estoy jugando (ABS_1)", "Tiendo a ponerme nervioso si no puedo jugar por alguna razón (ABS_3)" y "Creo que jugar ha puesto en peligro la relación con mi pareja (CON_3)".

En la figura 1 se presenta la media ideal que los estudiantes pueden llegar a adquirir para desarrollar el IGD. Asimismo, se expone la media totalizada de dicho trastorno así como la puntuación media obtenida en cada una de las etapas educativas. La media de las variables se sitúa entre 3 y 1.5, lo que muestra resultados medios-bajos. La mayoría de las variables se ubican por dejado de la media ideal, exceptuando "Sé que mis actividades cotidianas no se han visto negativamente afectadas por jugar (CON_4)" y "Creo que jugar está afectando negativamente áreas de mi vida (CON_5)", situándose por encima de la media (Figura 1).

Aquellas variables con menos media son "Creo que jugar se ha convertido en la actividad que más tiempo consume mi vida (PRO_3)", "Necesito pasar cada vez más tiempo jugando a videojuegos (TOL_2)” y "He mentido a mis familiares debido a la cantidad que dedico a videojuegos (CON_2)". 
Tabla 1

Descriptivo del nivel de competencia digital por áreas

\begin{tabular}{|c|c|c|c|c|c|c|c|c|c|}
\hline & \multicolumn{5}{|c|}{ Escala Likert $n / \%$} & \multicolumn{4}{|c|}{ Parámetros } \\
\hline & 1 & 2 & 3 & 4 & 5 & $M$ & DT & ASI & CUR \\
\hline \multicolumn{10}{|l|}{ PRO } \\
\hline & 67 & 39 & 38 & 27 & 9 & 2.29 & 1.248 & .534 & -.881 \\
\hline \multirow[t]{2}{*}{ PRO_1 } & 37.2 & 21.7 & 21.1 & 15 & 5 & & & & \\
\hline & 84 & 40 & 27 & 22 & 7 & 2.04 & 1.209 & .873 & -.406 \\
\hline \multirow[t]{2}{*}{ PRO_2 } & 46.7 & 22.2 & 15 & 12.2 & 3.9 & & & & \\
\hline & 98 & 39 & 19 & 20 & 4 & 1.85 & 1.131 & 1.143 & .147 \\
\hline PRO_3 & 54.4 & 21.7 & 10.6 & 11.1 & 2.2 & & & & \\
\hline \multicolumn{10}{|l|}{ ANI } \\
\hline & 36 & 31 & 44 & 25 & 44 & 3.06 & 1.448 & -.009 & -1.304 \\
\hline \multirow[t]{2}{*}{ ANI_1 } & 20 & 17.2 & 24.4 & 13.9 & 24.4 & & & & \\
\hline & 85 & 22 & 29 & 30 & 14 & 2.26 & 1.395 & .608 & -1.079 \\
\hline \multirow[t]{2}{*}{ ANI_2 } & 47.2 & 12.2 & 16.1 & 16.7 & 7.8 & & & & \\
\hline & 80 & 24 & 31 & 34 & 11 & 2.29 & 1.360 & .514 & -1.168 \\
\hline ANI_3 & 44.4 & 13.3 & 17.2 & 18.9 & 6.1 & & & & \\
\hline \multicolumn{10}{|l|}{ TOL } \\
\hline & 69 & 35 & 35 & 31 & 10 & 2.32 & 1.293 & .493 & -1.029 \\
\hline \multirow[t]{2}{*}{ TOL_1 } & 38.3 & 19.4 & 19.4 & 17.2 & 5.6 & & & & \\
\hline & 104 & 34 & 21 & 16 & 5 & 1.80 & 1.126 & 1.258 & .495 \\
\hline \multirow[t]{2}{*}{ TOL_2 } & 57.8 & 18.9 & 11.7 & 8.9 & 2.8 & & & & \\
\hline & 98 & 35 & 24 & 17 & 6 & 1.88 & 1.161 & 1.129 & .164 \\
\hline TOL_3 & 54.4 & 19.4 & 13.3 & 9.4 & 3.3 & & & & \\
\hline \multicolumn{10}{|l|}{$A B S$} \\
\hline & 99 & 34 & 19 & 19 & 9 & 1.92 & 1.237 & 1.146 & .080 \\
\hline \multirow[t]{2}{*}{ ABS_1 } & 55 & 18.9 & 10.6 & 10.6 & 5 & & & & \\
\hline & 103 & 30 & 21 & 18 & 8 & 1.88 & 1.217 & 1.176 & .157 \\
\hline \multirow[t]{2}{*}{ ABS_2 } & 57.2 & 16.7 & 11.7 & 10 & 4 & & & & \\
\hline & 105 & 26 & 22 & 20 & 7 & 1.88 & 1.222 & 1.128 & -.025 \\
\hline ABS_3 & 58.3 & 14.4 & 12.2 & 11.1 & 3.9 & & & & \\
\hline \multicolumn{10}{|l|}{ CON } \\
\hline & 90 & 28 & 38 & 21 & 3 & 1.99 & 1.155 & .736 & -.745 \\
\hline \multirow[t]{2}{*}{ CON_1 } & 50 & 15.6 & 21.1 & 11.7 & 1.7 & & & & \\
\hline & 113 & 22 & 18 & 25 & 2 & 1.78 & 1.159 & 1.150 & -.173 \\
\hline \multirow[t]{2}{*}{ CON_2 } & 62.8 & 12.2 & 10 & 13.9 & 1.1 & & & & \\
\hline & 101 & 34 & 14 & 23 & 8 & 1.91 & 1.245 & 1.147 & -.010 \\
\hline \multirow[t]{2}{*}{ CON_3 } & 56.1 & 18.9 & 7.8 & 12.8 & 4.4 & & & & \\
\hline & 56 & 19 & 35 & 32 & 38 & 2.87 & 1.539 & .049 & -1.476 \\
\hline \multirow[t]{2}{*}{ CON_4 } & 31.1 & 10.6 & 19.4 & 17.8 & 21.1 & & & & \\
\hline & 50 & 25 & 34 & 34 & 37 & 2.91 & 1.505 & .033 & -1.433 \\
\hline CON_5 & 27.8 & 13.9 & 18.9 & 18.9 & 20.6 & & & & \\
\hline \multicolumn{10}{|l|}{ REC } \\
\hline & 85 & 37 & 32 & 15 & 11 & 2.06 & 1.240 & .941 & -.191 \\
\hline REC_1 & 47.2 & 20.6 & 17.8 & 8.3 & 6.1 & & & & \\
\hline & 100 & 23 & 32 & 17 & 8 & 1.94 & 1.231 & .996 & -.237 \\
\hline REC_2 & 55.6 & 12.8 & 17.8 & 9.4 & 4.4 & & & & \\
\hline & 98 & 32 & 24 & 20 & 6 & 1.91 & 1.193 & 1.053 & -.114 \\
\hline REC 3 & 54.4 & 17.8 & 13.3 & 11.1 & 3.3 & & & & \\
\hline
\end{tabular}

Fuente: elaboración propia 
Figura 1

Distribución de las puntuaciones medias

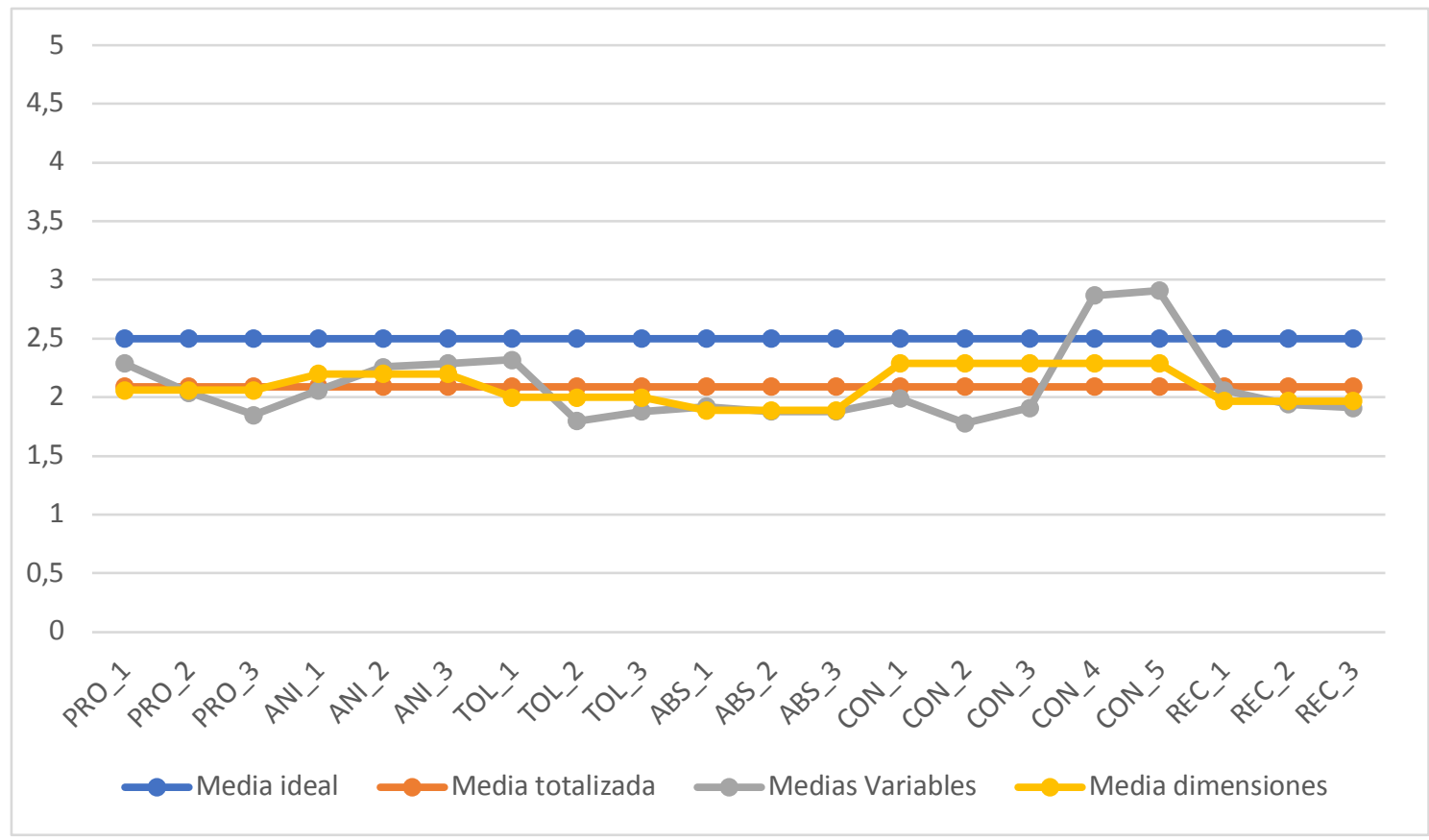

Fuente: elaboración propia

\subsection{Correlación entre el género y las variables IGC-20}

En el análisis se ve que hay contraste en muchas de las medias de las variables analizadas relacionadas con las valoraciones dadas por el género masculino y el femenino. En la mayoría de las variables son los hombres los que suelen presentar respuestas más altas que las mujeres. La variable más valorada es "Nunca juego a videojuegos para sentirme mejor (ANI_1)".

La variable más valorada por los hombres es "Sé que mis actividades cotidianas no se han visto negativamente afectadas por jugar (CON_4)" y la menos "He mentido a mis familiares debido a la cantidad de tiempo que dedico a videojuegos (CON_2)". La más valorada por las mujeres es "Nunca juego a videojuegos para sentirme mejor (ANI_1)" y la menos valorada es "He mentido a mis familiares debido a la cantidad de tiempo que dedico a videojuegos (CON 2)".

La curtosis y la asimetría exponen que la distribución de respuesta de las mujeres no es normal mientras que la de los hombres si lo es. La mayoría de las variables muestran una curtosis platicúrtica, aunque en menor porción también hay de tipo leptocúrtica y mesocúrtica (tabla 2).

Con respecto a los valores mostrados en el análisis correlacional entre género y PRO, se observa que existe una relación de significancia en "Suelo pensar en mi próxima sesión de juego cuando no estoy jugando (PRO_2)" y "Creo que jugar se ha convertido en la actividad que más tiempo consume mi vida (PRO_3)" pero no hay dicha relación en "A menudo pierdo horas de sueño debido a largas sesiones de juego (PRO_1)". La fuerza de asociación, según la $V$ de Cramer, en las variables de estudio donde hay relación de significancia es media-baja (tabla 3). 
Tabla 2

Estadísticos descriptivos entre hombres y mujeres de IGC-20.

\begin{tabular}{|c|c|c|c|c|c|c|}
\hline Variables & G & $n$ & $M$ & DT & As & $\mathrm{Cu}$ \\
\hline \multirow[t]{2}{*}{ PRO_1 } & $\mathrm{H}$ & 85 & 2.48 & 1.259 & .334 & -1.032 \\
\hline & $M$ & 95 & 2.12 & 1.219 & .745 & -.572 \\
\hline \multirow[t]{2}{*}{ PRO_2 } & $\mathrm{H}$ & 85 & 2.36 & 1.290 & .512 & -.990 \\
\hline & $M$ & 95 & 1.76 & 1.059 & 1.271 & .725 \\
\hline \multirow[t]{2}{*}{ PRO_3 } & $\mathrm{H}$ & 85 & 2.15 & 1.239 & .663 & -.825 \\
\hline & $\mathrm{M}$ & 95 & 1.58 & .952 & 1.773 & 2.447 \\
\hline \multirow[t]{2}{*}{ ANI_1 } & $\mathrm{H}$ & 85 & 2.92 & 1.338 & .062 & -1.129 \\
\hline & $M$ & 95 & 3.18 & 1.537 & -.110 & -1.429 \\
\hline \multirow[t]{2}{*}{ ANI_2 } & $\mathrm{H}$ & 85 & 2.69 & 1.439 & .164 & -1.357 \\
\hline & $M$ & 95 & 1.86 & 1.234 & 1.098 & -.202 \\
\hline \multirow[t]{2}{*}{ ANI_3 } & $\mathrm{H}$ & 85 & 2.72 & 1.385 & .085 & -1.308 \\
\hline & $M$ & 95 & 1.91 & 1.221 & .972 & -.511 \\
\hline \multirow[t]{2}{*}{ TOL_1 } & $\mathrm{H}$ & 85 & 2.64 & 1.387 & .247 & -1.284 \\
\hline & $\mathrm{M}$ & 95 & 2.04 & 1.138 & .623 & -.921 \\
\hline \multirow[t]{2}{*}{ TOL_2 } & $\mathrm{H}$ & 85 & 2.05 & 1.234 & .805 & -.620 \\
\hline & $M$ & 95 & 1.58 & .974 & 1.855 & 2.924 \\
\hline \multirow[t]{2}{*}{ TOL_3 } & $\mathrm{H}$ & 85 & 2.14 & 1.236 & .694 & -.776 \\
\hline & $M$ & 95 & 1.64 & 1.041 & 1.693 & 2.213 \\
\hline \multirow[t]{2}{*}{ ABS_1 } & $\mathrm{H}$ & 85 & 2.28 & 1.342 & .616 & -.951 \\
\hline & $M$ & 95 & 1.59 & 1.037 & 1.893 & 2.862 \\
\hline \multirow[t]{2}{*}{ ABS_2 } & $\mathrm{H}$ & 85 & 2.24 & 1.403 & .655 & -1.012 \\
\hline & $M$ & 95 & 1.56 & .919 & 1.757 & 2.544 \\
\hline \multirow[t]{2}{*}{ ABS_3 } & $\mathrm{H}$ & 85 & 2.22 & 1.294 & .616 & -.849 \\
\hline & $\mathrm{M}$ & 95 & 1.57 & 1.068 & 1.849 & 2.228 \\
\hline \multirow[t]{2}{*}{ CON_1 } & $\mathrm{H}$ & 85 & 2.29 & 1.223 & .373 & -1.045 \\
\hline & $M$ & 95 & 1.73 & 1.026 & 1.119 & -.119 \\
\hline \multirow[t]{2}{*}{ CON_2 } & $\mathrm{H}$ & 85 & 2.07 & 1.261 & .667 & -1.153 \\
\hline & $M$ & 95 & 1.53 & .998 & 1.794 & 2.053 \\
\hline \multirow[t]{2}{*}{ CON_3 } & $\mathrm{H}$ & 85 & 2.28 & 1.385 & .576 & -1.206 \\
\hline & $M$ & 95 & 1.57 & .996 & 1.950 & 3.367 \\
\hline \multirow[t]{2}{*}{ CON_4 } & $\mathrm{H}$ & 85 & 2.74 & 1.416 & .112 & -1.311 \\
\hline & $M$ & 95 & 2.99 & 1.641 & -.042 & -1.614 \\
\hline \multirow[t]{2}{*}{ CON_5 } & $\mathrm{H}$ & 85 & 2.78 & 1.357 & .155 & -1.178 \\
\hline & $\mathrm{M}$ & 95 & 3.02 & 1.624 & -.096 & -1.595 \\
\hline \multirow[t]{2}{*}{ REC_1 } & $\mathrm{H}$ & 85 & 2.36 & 1.335 & .590 & -.823 \\
\hline & $M$ & 95 & 1.78 & 1.084 & 1.326 & .980 \\
\hline \multirow[t]{2}{*}{ REC_2 } & $\mathrm{H}$ & 85 & 2.29 & 1.308 & .578 & -.858 \\
\hline & $M$ & 95 & 1.63 & 1.072 & 1.526 & 1.210 \\
\hline \multirow[t]{2}{*}{ REC_3 } & $\mathrm{H}$ & 85 & 2.18 & 1.311 & .737 & -.773 \\
\hline & $M$ & 95 & 1.67 & 1.026 & 1.361 & .766 \\
\hline
\end{tabular}


Tabla 3

Correlación entre género y PRO

\begin{tabular}{|c|c|c|c|c|c|c|c|c|c|}
\hline \multirow{2}{*}{ PRO } & \multicolumn{5}{|c|}{ Escala Likert $n(\%)$} & \multicolumn{4}{|c|}{ Parámetros } \\
\hline & 1 & 2 & 3 & 4 & 5 & $\chi^{2}(g l)$ & $p$-va. & $\mathrm{V}$ & Cont \\
\hline PRO_1 & & & & & & $4.835(4)$ & .305 & .164 & .162 \\
\hline Hombre & $25(29.4)$ & $20(23.5)$ & $19(22.4)$ & $16(18.8)$ & $5(5.9)$ & & & & \\
\hline Mujer & $42(44.2)$ & $19(20)$ & $19(20)$ & 11(11.6) & $4(4.2)$ & & & & \\
\hline PRO_2 & & & & & & $13.803(4)$ & .008 & .277 & .267 \\
\hline Hombre & $29(34.1)$ & $22(25.9)$ & 13(15.3) & 16(18.8) & $5(5.9)$ & & & & \\
\hline Mujer & $55(57.9)$ & 18(18.9) & $14(14.7)$ & $5(6.3)$ & $2(2.1)$ & & & & \\
\hline PRO_3 & & & & & & $15.178(4)$ & .004 & .290 & .279 \\
\hline Hombre & $37(43.5)$ & $17(20)$ & $15(17.6)$ & $13(15.3)$ & $3(3.5)$ & & & & \\
\hline Mujer & $61(64.2)$ & $22(23.2)$ & $4(4.2)$ & $7(7.4)$ & $1(1.1)$ & & & & \\
\hline
\end{tabular}

Con respecto a los valores mostrados en el análisis correlacional efectuado entre el género y ANI (tabla 2), se aprecia que hay relación de significancia en las variables pertenecientes a ANI, las cuales son "Nunca juego a videojuegos para sentirme mejor (ANI_1)", "Juego a videojuegos porque me ayuda a lidiar con los sentimientos desagradables que puedo tener (ANI_2)" y "Juego a videojuegos para olvidar cosas que me preocupan (ANI_3)". En todas ellas, la fuerza de asociación, según la V de Cramer, resulta que dicha relación es media-baja (tabla 4).

Tabla 4

Correlación entre género y ANI

\begin{tabular}{|c|c|c|c|c|c|c|c|c|c|}
\hline \multirow{2}{*}{ ANI } & \multicolumn{5}{|c|}{ Escala Likert $n(\%)$} & \multicolumn{4}{|c|}{ Parámetros } \\
\hline & 1 & 2 & 3 & 4 & 5 & $\chi^{2}(g l)$ & $p$-va. & V & Cont \\
\hline ANI_1 & & & & & & $11.425(4)$ & .022 & .252 & .244 \\
\hline Hombre & $16(18.8)$ & $18(21.2)$ & $21(24.7)$ & $17(20)$ & $13(15.3)$ & & & & \\
\hline Mujer & $20(21.1)$ & 13(13.7) & $23(24.2)$ & $8(8.4)$ & $31(32.6)$ & & & & \\
\hline ANI_2 & & & & & & $17.620(4)$ & .001 & .313 & .299 \\
\hline Hombre & $27(31.8)$ & $12(14.1)$ & $17(20)$ & $18(21.2)$ & $11(12.9)$ & & & & \\
\hline Mujer & $58(61.1)$ & $10(10.5)$ & $12(12.6)$ & $12(12.6)$ & $3(3.2)$ & & & & \\
\hline ANI_3 & & & & & & $17.844(4)$ & .001 & .315 & .300 \\
\hline Hombre & $25(29.4)$ & $12(14.1)$ & $19(22.4)$ & $20(23.5)$ & $9(10.6)$ & & & & \\
\hline Mujer & $55(57.9)$ & $12(12.6)$ & $12(12.6)$ & $14(14.7)$ & $2(2.1)$ & & & & \\
\hline
\end{tabular}

Fuente: elaboración propia

En la correlación entre el género y TOL, se observa una relación de significancia en todas las variables de la dimensión TOL, destacando "Durante el último año he aumentado significativamente la cantidad de horas de juego a videojuegos (TOL_1)”, "Necesito pasar cada vez más tiempo jugando a videojuegos (TOL_2)”, "A menudo pienso que un día entero no es suficiente para hacer todo lo necesito hacer en el juego. (TOL_3)". La fuerza que existe en dicha asociación, según la V de Cramer, es media-baja (tabla 5).

En la correlación entre género y $A B S$, se muestra una relación de significancia en todas las variables de la dimensión $A B S$, es decir, "Me siento más irritable cuando no estoy jugando (ABS_1)", "Me siento triste si no puedo jugar a videojuegos (ABS_2)" y "Tiendo a ponerme nervioso si no puedo jugar por alguna razón (ABS_3)". En dicha relación la fuerza de asociación, según la $\vee$ de Cramer en dichas variables, es medio-baja (tabla 6). 
Tabla 5

Correlación entre género y TOL

\begin{tabular}{|c|c|c|c|c|c|c|c|c|c|}
\hline \multirow{2}{*}{ TOL } & \multicolumn{5}{|c|}{ Escala Likert $n$ (\%) } & \multicolumn{4}{|c|}{ Parámetros } \\
\hline & 1 & 2 & 3 & 4 & 5 & $\chi^{2}(g l)$ & $p$-va. & $\mathrm{V}$ & Cont \\
\hline TOL_1 & & & & & & $14.129(4)$ & .007 & .280 & .270 \\
\hline Hombre & $25(29.4)$ & $18(21.2)$ & $14(16.5)$ & $19(22.4)$ & $9(10.6)$ & & & & \\
\hline Mujer & $44(46.3)$ & $17(17.9)$ & $21(22.1)$ & $12(12.6)$ & $1(1.1)$ & & & & \\
\hline TOL_2 & & & & & & $10.690(4)$ & .030 & .244 & .237 \\
\hline Hombre & $42(49.4)$ & $14(16.5)$ & $15(17.6)$ & $11(12.9)$ & $3(3.5)$ & & & & \\
\hline Mujer & $62(65.3)$ & $20(21.1)$ & $6(6.3)$ & $5(5.3)$ & $2(2.1)$ & & & & \\
\hline TOL_3 & & & & & & $10.815(4)$ & .029 & .245 & .238 \\
\hline Hombre & $37(43.5)$ & $18(21.2)$ & $14(16.5)$ & $13(15.3)$ & $3(3.5)$ & & & & \\
\hline Mujer & $61(64.2)$ & $17(17.9)$ & $10(10.5)$ & $4(4.2)$ & $3(3.2)$ & & & & \\
\hline
\end{tabular}

Fuente: elaboración propia

Tabla 6

Correlación entre género y ABS

\begin{tabular}{|c|c|c|c|c|c|c|c|c|c|}
\hline \multirow{2}{*}{ ABS } & \multicolumn{5}{|c|}{ Escala Likert $n$ (\%) } & \multicolumn{4}{|c|}{ Parámetros } \\
\hline & 1 & 2 & 3 & 4 & 5 & $\chi^{2}(g l)$ & $p$-va. & V & Cont \\
\hline ABS_1 & & & & & & $15.830(4)$ & .003 & .297 & .284 \\
\hline Hombre & $35(41.2)$ & $17(20)$ & $13(15.3)$ & $14(16.5)$ & $6(7.1)$ & & & & \\
\hline Mujer & $64(67.4)$ & 17(17.9) & $6(6.3)$ & $5(5.3)$ & $3(3.2)$ & & & & \\
\hline ABS_2 & & & & & & $17.502(4)$ & .002 & .312 & .298 \\
\hline Hombre & $41(48.2)$ & $10(11.8)$ & $14(16.5)$ & $13(15.3)$ & $7(8.2)$ & & & & \\
\hline Mujer & $62(65.3)$ & $20(21.1)$ & $7(7.4)$ & $5(5.3)$ & $1(1.1)$ & & & & \\
\hline ABS_3 & & & & & & $21.786(4)$ & .000 & .348 & .329 \\
\hline Hombre & $37(43.5)$ & $13(15.3)$ & $19(22.4)$ & $11(12.9)$ & $5(5.9)$ & & & & \\
\hline Mujer & $68(71,6)$ & $13(13.7)$ & $3(3.2)$ & $9(9.5)$ & $2(2.1)$ & & & & \\
\hline
\end{tabular}

En la correlación entre género y CON, existe una relación de significancia de todas las variables de la dimensión CON, destacando "He perdido interés en otras aficiones debido a jugar (CON_1)", "He mentido a mis familiares debido a la cantidad de tiempo que dedico a videojuegos (CON_2)", "Creo que jugar ha puesto en peligro la relación con mi pareja (CON_3)", "Sé que mis actividades cotidianas no se han visto negativamente afectadas por jugar (CON_4)" y “Creo que jugar está afectando negativamente áreas importantes de mi vida (CON_5)". La fuerza de relación es media-baja, según el estadístico de V de Cramer (tabla 7).

En la correlación entre género y REC, en las variables de "Me gustaría reducir mi tiempo de juego pero me resulta difícil (REC_1)" y "No creo que pudiera dejar de jugar (REC_2)" existe una relación de significancia, mientras que en "A menudo me propongo jugar menos pero acabo por lo lograrlo (REC_3)" no la hay. Según la V de Cramer, la fuerza de asociación media-baja (tabla 8). 
Tabla 7

Correlación entre género y CON

\begin{tabular}{|c|c|c|c|c|c|c|c|c|c|}
\hline \multirow{2}{*}{ CON } & \multicolumn{5}{|c|}{ Escala Likert $n(\%)$} & \multicolumn{4}{|c|}{ Parámetros } \\
\hline & 1 & 2 & 3 & 4 & 5 & $\chi^{2}(g l)$ & $p$-va. & V & Cont \\
\hline CON_1 & & & & & & $13.676(4)$ & .008 & .276 & .266 \\
\hline Hombre & $33(38.8)$ & $12(14.1)$ & $25(29.4)$ & $12(14.1)$ & $3(3.5)$ & & & & \\
\hline Mujer & $57(60)$ & $16(16.8)$ & 13(13.7) & $9(9.5)$ & $0(0)$ & & & & \\
\hline CON_2 & & & & & & $11.721(4)$ & .020 & .255 & .247 \\
\hline Hombre & $43(50.6)$ & $13(15.3)$ & $10(11.8)$ & $18(21.2)$ & $1(1.2)$ & & & & \\
\hline Mujer & $70(73.7)$ & $9(9.5)$ & $8(8.4)$ & $7(7.4)$ & $1(1.1)$ & & & & \\
\hline CON_3 & & & & & & $20.075(4)$ & .000 & .334 & .317 \\
\hline Hombre & $37(43.5)$ & $17(20)$ & $6(7.1)$ & $20(23.5)$ & $5(5.9)$ & & & & \\
\hline Mujer & $64(67.4)$ & 17(17.9) & $8(8.4)$ & $3(3.2)$ & $3(3.2)$ & & & & \\
\hline CON_4 & & & & & & $8.925(4)$ & .063 & .223 & .217 \\
\hline Hombre & $25(29.4)$ & $12(14.1)$ & $19(22.4)$ & $18(21.2)$ & $11(12.9)$ & & & & \\
\hline Mujer & $31(32.6)$ & $7(7.4)$ & $16(16.8)$ & $14(14.7)$ & $27(28.4)$ & & & & \\
\hline CON_5 & & & & & & $12.876(4)$ & .012 & .267 & .258 \\
\hline Hombre & $20(23.5)$ & $18(21.2)$ & $19(22.4)$ & $17(20)$ & $11(12.9)$ & & & & \\
\hline Mujer & $30(31.6)$ & $7(7.4)$ & $15(15.8)$ & 17(17.9) & $26(27.4)$ & & & & \\
\hline
\end{tabular}

Fuente: elaboración propia

Tabla 8

Correlación entre género y REC

\begin{tabular}{|c|c|c|c|c|c|c|c|c|c|}
\hline \multirow{2}{*}{ REC } & \multicolumn{5}{|c|}{ Escala Likert $n$ (\%) } & \multicolumn{4}{|c|}{ Parámetros } \\
\hline & 1 & 2 & 3 & 4 & 5 & $\chi^{2}(g l)$ & $p$-va. & $\mathrm{V}$ & Cont \\
\hline REC_1 & & & & & & $10.166(4)$ & .038 & .238 & .231 \\
\hline Hombre & $31(36.5)$ & $18(21.2)$ & $18(21.2)$ & $10(11.8)$ & $8(9.4)$ & & & & \\
\hline Mujer & $54(56.8)$ & $19(20)$ & $14(14.7)$ & $5(5.3)$ & $3(3.2)$ & & & & \\
\hline REC_2 & & & & & & $16.461(4)$ & .002 & .302 & .289 \\
\hline Hombre & $34(40)$ & 15(17.6) & $19(22.4)$ & $11(12.9)$ & $6(7.1)$ & & & & \\
\hline Mujer & $66(69.5)$ & $8(8.4)$ & $13(13.7)$ & $6(6.3)$ & $2(2.1)$ & & & & \\
\hline REC_3 & & & & & & $9.003(4)$ & .061 & .224 & .218 \\
\hline Hombre & $38(44.7)$ & $17(20)$ & $12(14.1)$ & $13(15.3)$ & $5(5.9)$ & & & & \\
\hline Mujer & $60(63.2)$ & $15(15.8)$ & $12(12.6)$ & $7(7.4)$ & $1(1.1)$ & & & & \\
\hline
\end{tabular}

Fuente: elaboración propia

\section{Discusión y Conclusiones}

La intromisión de las TIC y del mundo de Internet en las vidas de las personas, ha modificado el modo en el que estas se relacionan, se divierten y se socializan entre ellas, siendo utilizadas para evadirse de la realidad, pudiéndose hacer de manera problemática o volviéndose adictos a las mismas (Bouna, Muhle, Kornhuber y Lenz, 2015; Adamas et al., 2019; Bonnaire et al., 2019; Gómez-García, Rodríguez-Jiménez \& Marín-Marín, 2019; Hui, Wu \& Pun, 2019; Kim et al., 2019; Kircaburun, Griffiths \& Billieux, 2019; Müller et al., 2019; Zhou et al., 2019; Chen et al., 2020). 
Esta posible adicción es denominada como IGD y se define como el uso excesivo de Internet, juego o teléfonos inteligentes durante mucho tiempo, llegando incluso a provocar efectos negativos en los propios sujetos y, al mismo tiempo, afectando a su entorno social y familiar (Eichenbaum et al., 2015; Lemmens \& Hendriks, 2016; González et al., 2018; Kim et al., 2018; Schneider et al., 2017; Borges et al., 2019; Jo et al., 2019; Chen et al., 2020).

El IGD, según diversos estudios, está más presente en el género masculino, existiendo una proporción entre hombres y mujeres de un 3:1, ya que ellos juegan mucho más a juegos en línea y ellas, la mayoría de veces, tienen mayor control ejecutivo, resistiendo a los síntomas de dicho trastorno (Dong et al., 2018; Fam, 2018; Paulus et al., 2017; Dong et al., 2019; Wang et al., 2019).

Los resultados que se han obtenido según los niveles de los formandos de acuerdo a la adicción al juego en Internet se sitúan en una zona media-baja, ya que todas las dimensiones se encuentran por debajo de 2.5 , menos las dimensiones "Sé que mis actividades cotidianas no se han visto negativamente afectadas por jugar (CON_4)" y "Creo que jugar está afectando negativamente áreas importantes de mi vida (CON_5)" que sobrepasan la media totalizada. Se debe saber que la variable con más media es "Nunca juego a videojuegos para sentirme mejor (ANI_1)", y con menos es "He mentido a mis familiares debido a la cantidad de tiempo que dedico a videojuegos (CON_2)". Seguido de la dimensión con más media es CON, mientras que la media menor es ABS.

Cuando se habla del género, se tiene que saber que el hombre es el que presenta valores más altos de adicción al juego que las mujeres (Fam, 2018; Dong et al, 2018; Dong et al, 2019; Wang et al, 2019). Según los resultados, se ve que la variable más valorada por el género masculino es "Sé que mis actividades cotidianas no se han visto negativamente afectadas por jugar (CON_4)" y la que menos es "He mentido a mis familiares debido a la cantidad de tiempo que dedico a videojuegos (CON_2)". Y, siguiendo los datos, aquella variable más destacada por el género femenino es "Nunca juego a videojuegos para sentirme mejor (ANI_1)" y la menos valorada "He mentido a mis familiares debido a la cantidad de tiempo que dedico a videojuegos (CON_2)".

En esta investigación se observa que existe la relación de significancia en todas las variables de dicho estudio, mostrando que hay una influencia real del género en el desarrollo de conductas adictivas.

En la dimensión PRO existe la relación de significancia en "Suelo pensar en mi próxima sesión de juego cuando no estoy jugando (PRO_2)" y "Creo que jugar se ha convertido en la actividad que más tiempo consume mi vida (PRO_3)". En otra de las dimensiones, la REC, la relación de significancia más variable se da en "Me gustaría reducir mi tiempo de juego pero me resulta difícil (REC_1)” y “No creo que pudiera dejar de jugar (REC_2)”.

En el resto de las dimensiones que son ANI, TOL, ABS y CON, en cada una de sus variables, muestran que existe relación de significancia. En todas las variables que hay dicha relación, se sabe que la relación de fuerza es mediabaja.

Se puede concluir indicando que el nivel de adicción al juego que presentan los estudiantes es bajo en la mayoría de las variables, siendo el género un factor influyente para que los estudiantes puedan tener una mayor predisposición a desarrollar factores adictivos.

La prospectiva de esta investigación es identificar los niveles de adicción al juego de los estudiantes, con la intención de ofrecer más datos a la comunidad científica y concienciar a la sociedad, y concretamente a las Administraciones Educativas, para que apliquen y desarrollen programas de formación y prevención específicos.

Las limitaciones del estudio se centran en varios elementos. Primero, la población de estudio tiene diversas peculiaridades y características específicas. Por ello se debe tener cuidado con los datos recogidos a otras poblaciones. Y por otro lado, no se ha podido aplicar técnicas de muestreo en dicho desarrollo, por lo que se ha 
realizado por conveniencia. El método y la recogida de datos ha sido un esfuerzo enorme para los investigadores acerca de la recogida de datos y la muestra de ellos en el estudio.

Las futuras líneas de investigación se centran en especificar, aún más, todo lo relacionado con el trastorno IGD en los estudiantes del ámbito universitario, asociándolo a otros aspectos educativos como puede ser la motivación, interés e implicación en la tarea.

\section{Referencias bibliográficas}

Adams, B., Stavropoulos, V., Burleigh, T., Liew, L., Beard, C., \& Griffiths, M. (2018). Internet Gaming Disorder Behaviors in Emergent Adulthood: a Pilot Study Examining the Interplay Between Anxiety and Family Cohesion. International Journal Of Mental Health And Addiction, 17(4), 828-844. doi:10.1007/s11469-0189873-0

Allen, J., \& Anderson, C. (2018). Satisfaction and frustration of basic psychological needs in the real world and in video games predict internet gaming disorder scores and well-being. Computers In Human Behavior, 84, 220-229. doi:10.1016/j.chb.2018.02.034

American Psychiatric Association. (2018). Dsm-5. manual diagnostico y estadistico de los trastornos mentales. ED Médica Panamericana.

Bonnaire, C., \& Baptista, D. (2019). Internet gaming disorder in male and female young adults: The role of alexithymia, depression, anxiety and gaming type. Psychiatry Research, 272, 521-530. doi:10.1016/j.psychres.2018.12.158

Bonnaire, C., Liddle, H., Har, A., Nielsen, P., \& Phan, O. (2019). Why and how to include parents in the treatment of adolescents presenting Internet gaming disorder?. Journal Of Behavioral Addictions, 8(2), 201-212. doi:10.1556/2006.8.2019.27

Borges, G., Orozco, R., Benjet, C., Martínez Martínez, K., Contreras, E., \& Jiménez Pérez, A. et al. (2019). DSM-5 Internet gaming disorder among a sample of Mexican first-year college students. Journal Of Behavioral Addictions, 8(4), 714-724. doi:10.1556/2006.8.2019.62

Bouna, P., Aufleger, B., Braun, S., Gattnar, M., Kallmayer, S., \& Wagner, H. et al. (2018). Cross-Sectional and Longitudinal Evaluation of the Social Network Use Disorder and Internet Gaming Disorder Criteria. Frontiers In Psychiatry, 9. doi:10.3389/fpsyt.2018.00692

Bouna, P., Mühle, C., Kornhuber, J., \& Lenz, B. (2015). Internet gaming disorder, social network disorder and laterality: handedness relates to pathological use of social networks. Journal Of Neural Transmission, 122(8), 1187-1196. doi:10.1007/s00702-014-1361-5

Cai, C., Yuan, K., Yin, J., Feng, D., Bi, Y., \& Li, Y. et al. (2016). Striatum morphometry is associated with cognitive control deficits and symptom severity in internet gaming disorder. Brain Imaging And Behavior, 10(1), 1220. doi:10.1007/s11682-015-9358-8

Carlisle, K., Neukrug, E., Pribesh, S., \& Krahwinkel, J. (2019). Personality, Motivation, and Internet Gaming Disorder: Conceptualizing the Gamer. Journal Of Addictions \& Offender Counseling, 40(2), 107-122. doi:10.1002/jaoc.12069

Chen, C., Yen, J., Wang, P., Liu, G., Yen, C., \& Ko, C. (2016). Altered Functional Connectivity of the Insula and Nucleus Accumbens in Internet Gaming Disorder: A Resting State fMRI Study. European Addiction Research, 22(4), 192-200. doi:10.1159/000440716 
Chen, I., Strong, C., Lin, Y., Tsai, M., Leung, H., \& Lin, C. et al. (2020). Time invariance of three ultra-brief internet-related instruments: Smartphone Application-Based Addiction Scale (SABAS), Bergen Social Media Addiction Scale (BSMAS), and the nine-item Internet Gaming Disorder Scale- Short Form (IGDS-SF9) (Study Part B). Addictive Behaviors, 101, 105960. doi:10.1016/j.addbeh.2019.04.018

Cheng, C., Cheung, M., \& Wang, H. (2018). Multinational comparison of internet gaming disorder and psychosocial problems versus well-being: Meta-analysis of 20 countries. Computers In Human Behavior, 88, 153-167. doi:10.1016/j.chb.2018.06.033

Dang, D., Zhang, M., Leong, K., \& Wu, A. (2019). The Predictive Value of Emotional Intelligence for Internet Gaming Disorder: A 1-Year Longitudinal Study. International Journal Of Environmental Research And Public Health, 16(15), 1-14. doi:10.3390/ijerph16152762

Dong, G., Zheng, H., Liu, X., Wang, Y., Du, X., \& Potenza, M. (2018). Gender-related differences in cue-elicited cravings in Internet gaming disorder: The effects of deprivation. Journal Of Behavioral Addictions, 7(4), 953-964. doi:10.1556/2006.7.2018.118

Du, X., Qi, X., Yang, Y., Du, G., Gao, P., \& Zhang, Y. et al. (2016). Altered Structural Correlates of Impulsivity in Adolescents with Internet Gaming Disorder. Frontiers In Human Neuroscience, 10. doi:10.3389/fnhum.2016.00004

Eichenbaum, A., Kattner, F., Bradford, D., Gentile, D., \& Green, C. (2015). Role-Playing and Real-Time Strategy Games Associated with Greater Probability of Internet Gaming Disorder. Cyberpsychology, Behavior, And Social Networking, 18(8), 480-485. doi:10.1089/cyber.2015.0092

Evren, C., Dalbudak, E., Topcu, M., Kutlu, N., Evren, B., \& Pontes, H. (2018). Psychometric validation of the Turkish nine-item Internet Gaming Disorder Scale-Short Form (IGDS9-SF). Psychiatry Research, 265, 349354. doi:10.1016/j.psychres.2018.05.002

Fam, J. (2018). Prevalence of internet gaming disorder in adolescents: A meta-analysis across three decades. Scandinavian Journal Of Psychology, 59(5), 524-531. doi:10.1111/sjop.12459

Fuster, H., Carbonell, X., Pontes, H., \& Griffiths, M. (2016). Spanish validation of the Internet Gaming Disorder20 (IGD-20) Test. Computers In Human Behavior, 56, 215-224. doi:10.1016/j.chb.2015.11.050

Gómez, R., Stavropoulos, V., Beard, C., \& Pontes, H. (2018). Item Response Theory Analysis of the Recoded Internet Gaming Disorder Scale-Short-Form (IGDS9-SF). International Journal Of Mental Health And Addiction, 17(4), 859-879. doi:10.1007/s11469-018-9890-z

Gómez-García, G., Rodríguez-Jiménez, C., \& Marín-Marín, J. (2019). La trascendencia de la Realidad Aumentada en la motivación estudiantil. Una revisión sistemática y meta-análisis. Alteridad, 15(1), 36-46. doi: 10.17163/alt.v15n1.2020.03

González-Bueso, V., Santamaría, J., Fernández, D., Merino, L., Montero, E., \& Jiménez-Murcia, S. et al. (2018). Internet Gaming Disorder in Adolescents: Personality, Psychopathology and Evaluation of a Psychological Intervention Combined With Parent Psychoeducation. Frontiers In Psychology, 9. doi:10.3389/fpsyg.2018.00787

Griffiths, M., Rooij, A., Kardefelt-Winther, D., Starcevic, V., Király, O., \& Pallesen, S. et al. (2015). Working towards an international consensus on criteria for assessing internet gaming disorder: a critical commentary on Petry et al . (2014). Addiction, 111(1), 167-175. doi:10.1111/add.13057 
Han, D., Yoo, M., Renshaw, P., \& Petry, N. (2018). A cohort study of patients seeking Internet gaming disorder treatment. Journal Of Behavioral Addictions, 7(4), 930-938. doi:10.1556/2006.7.2018.102

Hernández, R., Fernández, C., y Baptista, M.P. (2014). Metodología de la investigación. Madrid, Spain: McGraw Hill.

Hsieh, D.L., y Hsiao, T.C. (2016). Heart Rate Variability of Internet Gaming Disorder Addicts in Emotional States. 2016 3을 International conference on biomedical engineering (BME-HUST), 155-158.

Hui, B., S. Wu, A., \& Pun, N. (2019). Disentangling the effects of empathy components on Internet gaming disorder: A study of vulnerable youth in China. Journal Of Behavioral Addictions, 8(1), 181-189. doi:10.1556/2006.8.2019.12

Hui, B., Wu, A., Siu, N., Chung, M., \& Pun, N. (2019). The Effects of Need Satisfaction and Dissatisfaction on Flourishing among Young Chinese Gamers: The Mediating Role of Internet Gaming Disorder. International Journal Of Environmental Research And Public Health, 16(22), 4367. doi:10.3390/ijerph16224367

Jeong, H., Yim, H., Lee, S., Lee, H., Potenza, M., Jo, S., \& Son, H. (2019). Reciprocal relationship between depression and Internet gaming disorder in children: A 12-month follow-up of the iCURE study using crosslagged path analysis. Journal Of Behavioral Addictions, 8(4), 725-732. doi:10.1556/2006.8.2019.74

Ji, H.M., Hsieh, D.L., y Hsiao, T.C. (2016). Heart Rate Variability of Internet Gaming Disorder Addicts in Emotional States. 3 International conference on biomedical engineering (BME-HUST), 150-154.

Jo, Y., Bhang, S., Choi, J., Lee, H., Lee, S., \& Kweon, Y. (2019). Clinical Characteristics of Diagnosis for Internet Gaming Disorder: Comparison of DSM-5 IGD and ICD-11 GD Diagnosis. Journal Of Clinical Medicine, 8(7), 945. doi:10.3390/jcm8070945

Kang, K., Jung, T., Park, I., \& Han, D. (2018). Effects of Equine-Assisted Activities and Therapies on the Affective Network of Adolescents with Internet Gaming Disorder. The Journal Of Alternative And Complementary Medicine, 24(8), 841-849. doi:10.1089/acm.2017.0416

Kaptsis, D., King, D., Delfabbro, P., \& Gradisar, M. (2016). Withdrawal symptoms in internet gaming disorder: A systematic review. Clinical Psychology Review, 43, 58-66. doi:10.1016/j.cpr.2015.11.006

Kim, J., Chun, J., Park, C., Cho, H., Choi, J., \& Yang, S. et al. (2019). The Correlation between the Frontostriatal Network and Impulsivity in Internet Gaming Disorder. Scientific Reports, 9(1). doi:10.1038/s41598-01837702-4

Kim, M., Jung, Y., Kyeong, S., Shin, Y., Kim, E., \& Kim, J. (2018). Neural Correlates of Distorted Self-concept in Individuals With Internet Gaming Disorder: A Functional MRI Study. Frontiers In Psychiatry, 9. doi:10.3389/fpsyt.2018.00330

Kim, M., Lee, T., Choi, J., Kwak, Y., Hwang, W., \& Kim, T. et al. (2019). Dysfunctional attentional bias and inhibitory control during anti-saccade task in patients with internet gaming disorder: An eye tracking study. Progress In Neuro-Psychopharmacology And Biological Psychiatry, 95, 109717. doi:10.1016/j.pnpbp.2019.109717

Kim, N., Hwang, S., Choi, J., Kim, D., Demetrovics, Z., \& Király, O. et al. (2016). Characteristics and Psychiatric Symptoms of Internet Gaming Disorder among Adults Using Self-Reported DSM-5 Criteria. Psychiatry Investigation, 13(1), 58. doi:10.4306/pi.2016.13.1.58 
King, D., Delfabbro, P., Perales, J., Deleuze, J., Király, O., Krossbakken, E., \& Billieux, J. (2019). Maladaptive player-game relationships in problematic gaming and gaming disorder: A systematic review. Clinical Psychology Review, 73, 101777. doi:10.1016/j.cpr.2019.101777

Kircaburun, K., Demetrovics, Z., Griffiths, M., Király, O., Kun, B., \& Tosuntaş, Ş. (2019). Trait Emotional Intelligence and Internet Gaming Disorder Among Gamers: The Mediating Role of Online Gaming Motives and Moderating Role of Age Groups. International Journal Of Mental Health And Addiction. doi:10.1007/s11469-019-00179-x

Kircaburun, K., Griffiths, M., \& Billieux, J. (2019). Psychosocial factors mediating the relationship between childhood emotional trauma and internet gaming disorder: a pilot study. European Journal Of Psychotraumatology, 10(1), 1565031. doi:10.1080/20008198.2018.1565031

Ko, C., Chen, S., Wang, C., Tsai, W., \& Yen, J. (2019). The Clinical Utility of the Chen Internet Addiction ScaleGaming Version, for Internet Gaming Disorder in the DSM-5 among Young Adults. International Journal Of Environmental Research And Public Health, 16(21), 4141. doi:10.3390/ijerph16214141

Ko, C., Lin, H., Lin, P., \& Yen, J. (2019). Validity, functional impairment and complications related to Internet gaming disorder in the DSM-5 and gaming disorder in the ICD-11. Australian \& New Zealand Journal Of Psychiatry, 000486741988149. doi:10.1177/0004867419881499

Kuss, D., Pontes, H., \& Griffiths, M. (2018). Neurobiological Correlates in Internet Gaming Disorder: A Systematic Literature Review. Frontiers In Psychiatry, 9. doi:10.3389/fpsyt.2018.00166

Laier, C., Wegmann, E., \& Brand, M. (2018). Personality and Cognition in Gamers: Avoidance Expectancies Mediate the Relationship Between Maladaptive Personality Traits and Symptoms of Internet-Gaming Disorder. Frontiers In Psychiatry, 9. doi:10.3389/fpsyt.2018.00304

Lee, T., Kim, M., Kwak, Y., Hwang, W., Kim, T., Choi, J., \& Kwon, J. (2018). Altered Eye-Movement Patterns During Text Reading in Obsessive-Compulsive Disorder and Internet Gaming Disorder. Frontiers In Behavioral Neuroscience, 12. doi:10.3389/fnbeh.2018.00248

Lemmens, J., \& Hendriks, S. (2016). Addictive Online Games: Examining the Relationship Between Game Genres and Internet Gaming Disorder. Cyberpsychology, Behavior, And Social Networking, 19(4), 270-276. doi:10.1089/cyber.2015.0415

Leung, H., Pakpour, A., Strong, C., Lin, Y., Tsai, M., \& Griffiths, M. et al. (2020). Measurement invariance across young adults from Hong Kong and Taiwan among three internet-related addiction scales: Bergen Social Media Addiction Scale (BSMAS), Smartphone Application-Based Addiction Scale (SABAS), and Internet Gaming Disorder Scale-Short Form (IGDS-SF9) (Study Part A). Addictive Behaviors, 101, 105969. doi:10.1016/j.addbeh.2019.04.027

Li, A., Chau, C., \& Cheng, C. (2019). Development and Validation of a Parent-Based Program for Preventing Gaming Disorder: The Game Over Intervention. International Journal Of Environmental Research And Public Health, 16(11), 1984. doi:10.3390/ijerph16111984

Liu, J., Li, W., Zhou, S., Zhang, L., Wang, Z., \& Zhang, Y. et al. (2015). Functional characteristics of the brain in college students with internet gaming disorder. Brain Imaging And Behavior, 10(1), 60-67.

doi:10.1007/s11682-015-9364-x 
Liu, L., Yao, Y., Li, C., Zhang, J., Xia, C., \& Lan, J. et al. (2018). The Comorbidity Between Internet Gaming Disorder and Depression: Interrelationship and Neural Mechanisms. Frontiers In Psychiatry, 9. doi:10.3389/fpsyt.2018.00154

Loton, D., y Lubman, D.I. (2016). Just one more level: Identifying and addressing internet gaming disorder within primary care. Australian Family Physician, 45 (1-2), 48-52.

Marmet, S., Studer, J., Grazioli, V., \& Gmel, G. (2018). Bidirectional Associations Between Self-Reported Gaming Disorder and Adult Attention Deficit Hyperactivity Disorder: Evidence From a Sample of Young Swiss Men. Frontiers In Psychiatry, 9. doi:10.3389/fpsyt.2018.00649

Moreno-Guerrero, A.J. (2019). Estudio bibliométrico de la producción científica en Web of Science: Formación Profesional y blended learning. Pixel-Bit. Revista de Medios y Educación, 56, 149-168. doi: 10.12795/pixelbit.2019.i56.08

Müller, K., Beutel, M., Dreier, M., \& Wölfling, K. (2019). A clinical evaluation of the DSM-5 criteria for Internet Gaming Disorder and a pilot study on their applicability to further Internet-related disorders. Journal Of Behavioral Addictions, 8(1), 16-24. doi:10.1556/2006.7.2018.140

Park, M., Choi, J., Park, S., Lee, J., Jung, H., \& Sohn, B. et al. (2016). Dysfunctional information processing during an auditory event-related potential task in individuals with Internet gaming disorder. Translational Psychiatry, 6(1), e721-e721. doi:10.1038/tp.2015.215

Paulus, F., Ohmann, S., von Gontard, A., \& Popow, C. (2018). Internet gaming disorder in children and adolescents: a systematic review. Developmental Medicine \& Child Neurology, 60(7), 645-659. doi:10.1111/dmcn.13754

Paulus, F., Sinzig, J., Mayer, H., Weber, M., \& von Gontard, A. (2017). Computer Gaming Disorder and ADHD in Young Children-a Population-Based Study. International Journal Of Mental Health And Addiction, 16(5), 1193-1207. doi:10.1007/s11469-017-9841-0

Pontes, H., \& Griffiths, M. (2016). Portuguese Validation of the Internet Gaming Disorder Scale-ShortForm. Cyberpsychology, Behavior, And Social Networking, 19(4), 288-293. doi:10.1089/cyber.2015.0605

Pontes, H.M., Schivinski, B., Brzozowska-Woś, M., \& Stavropoulos, V. (2019). Laxer Clinical Criteria for Gaming Disorder May Hinder Future Efforts to Devise an Efficient Diagnostic Approach: A Tree-Based Model Study. Journal Of Clinical Medicine, 8(10), 1730. doi:10.3390/jcm8101730

Rodríguez-García, A.M., Moreno-Guerrero, A.J., \& López, J. (2020). Nomophobia: An Individual's Growing Fear of Being without a Smartphone-A Systematic Literature Review. International Journal of Environmental Research and Public Health, 17(2), 1-19. doi: 10.3390/ijerph17020580

Romero-Rodríguez, J., Marín-Marín, J., Hinojo-Lucena, F., \& Gómez-García, G. (2021). An Explanatory Model of Problematic Internet Use of Southern Spanish University Students. Social Science Computer Review, 089443932199865. doi: 10.1177/0894439321998650

Schneider, L., King, D., \& Delfabbro, P. (2017). Maladaptive Coping Styles in Adolescents with Internet Gaming Disorder Symptoms. International Journal Of Mental Health And Addiction, 16(4), 905-916. doi:10.1007/s11469-017-9756-9 
Shin, D., Choi, A., Lee, J., Chung, S., Kim, B., \& Park, M. et al. (2019). The Mediating Effects of Affect on Associations between Impulsivity or Resilience and Internet Gaming Disorder. Journal Of Clinical Medicine, 8(8), 1102. doi:10.3390/jcm8081102

Sprong, M., Griffiths, M., Lloyd, D., Paul, E., \& Buono, F. (2019). Comparison of the Video Game Functional Assessment-Revised (VGFA-R) and Internet Gaming Disorder Test (IGD-20). Frontiers In Psychology, 10. doi:10.3389/fpsyg.2019.00310

Tabachnick, B.G., y Fidell, L.S. (2007). Estadísticas multivariadas (5Th ed.). Boston: Pearson.

Throuvala, M., Janikian, M., Griffiths, M., Rennoldson, M., \& Kuss, D. (2019). The role of family and personality traits in Internet gaming disorder: A mediation model combining cognitive and attachment perspectives. Journal Of Behavioral Addictions, 8(1), 48-62. doi:10.1556/2006.8.2019.05

Torres-Rodríguez, A., Griffiths, M., Carbonell, X., Farriols-Hernando, N., \& Torres-Jimenez, E. (2019). Internet Gaming Disorder Treatment: A Case Study Evaluation of Four Different Types of Adolescent Problematic Gamers. International Journal Of Mental Health And Addiction, 17(1), 1-12. doi:10.1007/s11469-017-98459

Trujillo-Torres, J., Hinojo-Lucena, M., Marín-Marín, J., Romero Díaz de la Guardia, J., \& Campos-Soto, A. (2014). Análisis de experiencias de aprendizajes basados en proyectos: prácticas colaborativas BLearning. EDMETIC, 4(1), 51. doi: 10.21071/edmetic.v4i1.2899

Wang, Q., Ren, H., Long, J., Liu, Y., \& Liu, T. (2019). Research progress and debates on gaming disorder. General Psychiatry, 32(3), e100071. doi:10.1136/gpsych-2019-100071

Wang, Z., Wu, L., Yuan, K., Hu, Y., Zheng, H., Du, X., \& Dong, G. (2018). Cortical thickness and volume abnormalities in Internet gaming disorder: Evidence from comparison of recreational Internet game users. European Journal Of Neuroscience, 48(1), 1654-1666. doi:10.1111/ejn.13987

Wartberg, L., Kriston, L., \& Thomasius, R. (2020). Internet gaming disorder and problematic social media use in a representative sample of German adolescents: Prevalence estimates, comorbid depressive symptoms and related psychosocial aspects. Computers In Human Behavior, 103, 31-36.

doi:10.1016/j.chb.2019.09.014

Yao, Y., Wang, L., Yip, S., Chen, P., Li, S., \& Xu, J. et al. (2015). Impaired decision-making under risk is associated with gaming-specific inhibition deficits among college students with Internet gaming disorder. Psychiatry Research, 229(1-2), 302-309. doi:10.1016/j.psychres.2015.07.004

Yen, J., Lin, H., Chou, W., Liu, T., \& Ko, C. (2019). Associations Among Resilience, Stress, Depression, and Internet Gaming Disorder in Young Adults. International Journal Of Environmental Research And Public Health, 16(17), 3181. doi:10.3390/ijerph16173181

Zajac, K., Ginley, M., \& Chang, R. (2019). Treatments of internet gaming disorder: a systematic review of the evidence. Expert Review Of Neurotherapeutics, 20(1), 85-93. doi:10.1080/14737175.2020.1671824

Zhang, J., Ma, S., Yip, S., Wang, L., Chen, C., \& Yan, C. et al. (2015). Decreased functional connectivity between ventral tegmental area and nucleus accumbens in Internet gaming disorder: evidence from resting state functional magnetic resonance imaging. Behavioral And Brain Functions, 11(1). doi:10.1186/s12993-0150082-8 